\title{
Univalence criteria for meromorphic functions and quasiconformal extensions
}

\author{
Murat Çağlar* and Halit Orhan
}

${ }^{*}$ Correspondence: mcaglar25@gmail.com

Department of Mathematics, Faculty of Science, Ataturk

University, Erzurum, 25240, Turkey

\begin{abstract}
The aim of this paper is to obtain sufficient conditions for univalence of meromorphic functions in the $\mathcal{U}^{*}$. Also, we refine a quasiconformal extension criterion with the help of Becker's method. A number of univalence conditions would follow upon specializing the parameters involved in our main results.
\end{abstract}

MSC: Primary 30C80; secondary 30C45; 30C62

Keywords: univalent function; meromorphic function; quasiconformal extension; univalence condition; Loewner chain

\section{Introduction}

We denote by $\mathcal{U}_{r}=\{z \in \mathbb{C}:|z|<r\}(0<r \leq 1)$ the disc of radius $r$ and let $\mathcal{U}=\mathcal{U}_{1}$. Let $\mathcal{A}$ denote the class of analytic functions in the open unit disc $\mathcal{U}$ which satisfy the usual normalization condition $f(0)=f^{\prime}(0)-1=0$. We denote by $\mathcal{S}$ the subclass of $\mathcal{A}$ consisting of functions $f(z)$ which are univalent in $\mathcal{U}$. Let $\Sigma$ denote the class of functions of the form $f(\zeta)=\zeta+\sum_{k=0}^{\infty} a_{k} \zeta^{-k}$ which are meromorphic in the exterior to the open unit disc $\mathcal{U}^{*}=\{\zeta \in \mathbb{C}:|\zeta|>1\}$ with a pole at the infinity residue 1 . We say that a sense-preserving homeomorphism $f$ of a plane domain $G \subset \mathbb{C}$ is $k$-quasiconformal if $f$ is absolutely continuous on almost all lines parallel to coordinate axes and $\left|f_{\bar{z}}\right| \leq k\left|f_{z}\right|$, almost everywhere in $G$, where $f_{\bar{z}}=\partial f / \partial \bar{z}, f_{z}=\partial f / \partial z$ and $k$ is a constant with $0 \leq k<1$.

In geometric function theory, the univalence of complex functions is an important property, but it is difficult, and in many cases impossible, to show directly that a certain complex function is univalent. For this reason, many authors found different types of sufficient conditions of univalence. One of the most important of these conditions of univalence in the domains $\mathcal{U}$ and the exterior of a closed unit disc is the well-known criterion of Becker $[1,2]$. Becker's work depends upon a clever use of the theory of Loewner chains and the generalized Loewner differential equation. Extensions of this criterion were given by Ahlfors [3], Lewandowski [4, 5], Miazga and Wesolowski [6], Ruscheweyh [7], and Singh and Chichra [8]. Also, the recent investigations on this subject by Deniz and Orhan [9-11], Ponnusamy and Sugawa [12], Răducanu et al. [13], Kanas and Lecko [14, 15] and Kanas and Srivastava [16].

In the present paper, firstly we study a number of new criteria for the univalence of the functions belonging to the class $\Sigma$. Finally, we obtain a refinement to a quasiconformal extension criterion of the main result. We also consider several interesting corollaries and consequences of our univalence criteria. Our considerations are based on the theory of Loewner chains.

(c) 2013 Çağlar and Orhan; licensee Springer. This is an Open Access article distributed under the terms of the Creative Commons Attribution License (http://creativecommons.org/licenses/by/2.0), which permits unrestricted use, distribution, and reproduction in any medium, provided the original work is properly cited. 


\section{Loewner chains and quasiconformal extensions}

Before proving our main theorem, we need a brief summary of the method of Loewner chains.

Let $\mathcal{L}(z, t)=a_{1}(t) z+a_{2}(t) z^{2}+\cdots, a_{1}(t) \neq 0$ be a function defined on $\mathcal{U} \times I$, where $I:=$ $[0, \infty)$ and $a_{1}(t)$ is a complex-valued, locally absolutely continuous function on $I . \mathcal{L}(z, t)$ is called a Loewner chain if $\mathcal{L}(z, t)$ satisfies the following conditions:

(i) $\mathcal{L}(z, t)$ is analytic and univalent in $\mathcal{U}$ for all $t \in I$,

(ii) $\mathcal{L}(z, t) \prec \mathcal{L}(z, s)$ for all $0 \leq t \leq s<\infty$,

where the symbol ' $\prec$ ' stands for subordination. If $a_{1}(t)=e^{t}$, then we say that $\mathcal{L}(z, t)$ is a standard Loewner chain.

In order to prove our main results, we need the following theorem due to Pommerenke [17] (also see [18]). This theorem is often used to find out univalency for an analytic function, apart from the theory of Loewner chains.

Theorem 2.1 (see Pommerenke [18]) Let $\mathcal{L}(z, t)=a_{1}(t) z+a_{2}(t) z^{2}+\cdots$ be analytic in $\mathcal{U}_{r}$ for all $t \in I$. Suppose that

(i) $\mathcal{L}(z, t)$ is a locally absolutely continuous function in the interval I, and locally uniform with respect to $\mathcal{U}_{r}$.

(ii) $a_{1}(t)$ is a complex-valued continuous function on I such that $a_{1}(t) \neq 0,\left|a_{1}(t)\right| \rightarrow \infty$ for $t \rightarrow \infty$ and

$$
\left\{\frac{\mathcal{L}(z, t)}{a_{1}(t)}\right\}_{t \in I}
$$

forms a normal family of functions in $\mathcal{U}_{r}$.

(iii) There exists an analytic function $p: \mathcal{U} \times I \rightarrow \mathbb{C}$ satisfying $\Re p(z, t)>0$ for all $z \in \mathcal{U}$, $t \in I$ and

$$
z \frac{\partial \mathcal{L}(z, t)}{\partial z}=p(z, t) \frac{\partial \mathcal{L}(z, t)}{\partial t}, \quad z \in \mathcal{U}_{r}, t \in I
$$

Then, for each $t \in I$, the function $\mathcal{L}(z, t)$ has an analytic and univalent extension to the whole disc $\mathcal{U}$ or the function $\mathcal{L}(z, t)$ is a Loewner chain.

Equation (2.1) is called the generalized Loewner differential equation.

The method of constructing quasiconformal extension criteria is based on the following result due to Becker (see [1, 2] and also [19]).

Theorem 2.2 Suppose that $\mathcal{L}(z, t)$ is a Loewner chain for which $p(z, t)$ in (2.1) satisfies the condition

$$
\begin{aligned}
p(z, t) & \in U(k):=\left\{w \in \mathbb{C}:\left|\frac{w-1}{w+1}\right| \leq k\right\} \\
& =\left\{w \in \mathbb{C}:\left|w-\frac{1+k^{2}}{1-k^{2}}\right| \leq \frac{2 k}{1-k^{2}}\right\} \quad(0 \leq k<1)
\end{aligned}
$$


for all $z \in \mathcal{U}$ and $t \geq 0$. Then $\mathcal{L}(z, t)$ admits a continuous extension to $\overline{\mathcal{U}}$ for each $t \geq 0$, and the function $F(z, \bar{z})$ defined by

$$
F(z, \bar{z})= \begin{cases}\mathcal{L}(z, 0), & |z|<1, \\ \mathcal{L}\left(\frac{z}{|z|}, \log |z|\right), & |z| \geq 1\end{cases}
$$

is a $k$-quasiconformal extension of $\mathcal{L}(z, 0)$ to $\mathbb{C}$.

Detailed information about Loewner chains and quasiconformal extension criteria can be found in [3, 20-22] and recently in [23-25].

\section{Univalence criteria}

Making use of Theorem 2.1, now we can prove our main result.

Theorem 3.1 Let $m \in \mathbb{R}^{+}$and s be a complex number such that $s=\alpha+i \beta, \alpha>0, \beta \in \mathbb{R}$; $1 / 2<m \leq \alpha$ and $f \in \Sigma, f^{\prime}(\zeta) \neq 0$. If there exists an analytic function $g$ in $\mathcal{U}^{*}$ such that $g(\zeta)=1+c_{2} \zeta^{-2}+\cdots$ and the inequalities

$$
\left|\frac{\zeta f^{\prime}(\zeta)}{f(\zeta) g(\zeta)}-\frac{m s}{\alpha}\right|<\frac{m|s|}{\alpha}
$$

and

$$
\left|\frac{\zeta f^{\prime}(\zeta)}{f(\zeta) g(\zeta)}-\left(2 \ln |\zeta| \frac{\zeta g^{\prime}(\zeta)}{g(\zeta)}+1\right) \frac{m s}{\alpha}\right| \leq \frac{m|s|}{\alpha}
$$

hold true for all $\zeta \in \mathcal{U}^{*}$, then the function $f$ is univalent in $\mathcal{U}^{*}$.

Proof We will prove that there exists a real number $r \in(0,1]$ such that the function $\mathcal{L}$ : $\mathcal{U}_{r} \times I \rightarrow \mathbb{C}$, defined formally by

$$
\mathcal{L}(z, t)=\frac{1}{f\left(e^{s t} / z\right)}\left\{e^{-2 m t g\left(e^{s t} / z\right)}\right\}^{-s}=a_{1}(t) z+a_{2}(t) z^{2}+\cdots,
$$

is analytic in $\mathcal{U}_{r}$ for all $t \in I$, where

$$
\begin{aligned}
& f\left(e^{s t} \zeta\right)=e^{s t} \zeta+b_{0}+b_{1} e^{-s t} \zeta^{-1}+\cdots \quad\left(\zeta \in \mathcal{U}^{*}\right), \\
& g\left(e^{s t} \zeta\right)=1+c_{2} e^{-2 s t} \zeta^{-2}+\cdots \quad\left(\zeta \in \mathcal{U}^{*}\right) .
\end{aligned}
$$

Since $g$ is analytic, the function

$$
\phi(z, t)=e^{-2 m \operatorname{tg}\left(e^{s t} / z\right)}
$$

is analytic in $\mathcal{U}$ and $\phi(0, t)=e^{-2 m t} \neq 0$. Then there exists a disc $\mathcal{U}_{r_{1}}, 0<r_{1} \leq 1$, in which $\phi(z, t) \neq 0$ for all $z \in \mathcal{U}_{r_{1}}$. We denote the uniform branch of $(\phi(z, t))^{-s}$ by $\phi_{1}$, which is equal to $e^{2 m s t}$ at origin. 
It follows from (3.3) that

$$
\mathcal{L}(z, t)=\frac{\phi_{1}(z, t)}{f\left(e^{s t} / z\right)}=a_{1}(t) z+a_{2}(t) z^{2}+\cdots
$$

and thus the function $\mathcal{L}(z, t)$ is analytic in $\mathcal{U}_{r_{1}}$.

We have

$$
a_{1}(t)=e^{s(2 m-1) t},
$$

for which we consider the uniform branch equal to $e^{2 m s t}$ at the origin.

Because $\alpha>0$ and $1 / 2<m \leq \alpha$, we have

$$
\lim _{t \rightarrow \infty}\left|a_{1}(t)\right|=\infty
$$

Moreover, $a_{1}(t) \neq 0$ for all $t \in I$.

After simple calculation, we obtain, for each $z \in \mathcal{U}$,

$$
\begin{aligned}
\lim _{t \rightarrow \infty} \frac{\mathcal{L}(z, t)}{a_{1}(t)} & =\frac{z}{e^{s(2 m-1) t}\left(e^{s t}+b_{0} z+b_{1} e^{-s t} z^{2}+\cdots\right)} \phi_{1}(z, t) \\
& =\frac{z}{\left(1+b_{0} e^{-s t} z+b_{1} e^{-2 s t} z^{2}+\cdots\right) e^{-2 m s t\left(c_{1} e^{-2 s t} z^{2}+\cdots\right)}} \\
& =z .
\end{aligned}
$$

The limit function $\psi(z)=z$ belongs to the family $\left\{\mathcal{L}(z, t) / a_{1}(t)\right\}$; then in every closed disc $\mathcal{U}_{r_{2}}, 0<r_{2}<r_{1}$, there exists a constant $K=K\left(r_{2}\right)$ such that

$$
\left|\frac{\mathcal{L}(z, t)}{a_{1}(t)}\right|<K, \quad \forall z \in \mathcal{U}_{r_{2}}, t \in I
$$

uniformly in this disc, provided that $t$ is sufficiently large. Then, by Montel's theorem, $\left\{\frac{\mathcal{L}(z, t)}{a_{1}(t)}\right\}_{t \in I}$ is a normal family in $\mathcal{U}_{r_{2}}$. From the analyticity of $\frac{\partial \mathcal{L}(z, t)}{\partial t}$, we obtain that for all fixed numbers $T>0$ and $r_{3}, 0<r_{3}<r_{2}$, there exists a constant $K_{1}>0$ (that depends on $T$ and $r_{3}$ ) such that

$$
\left|\frac{\partial \mathcal{L}(z, t)}{\partial t}\right|<K_{1}, \quad \forall z \in \mathcal{U}_{r_{3}}, t \in[0, T] .
$$

Therefore, the function $\mathcal{L}(z, t)$ is locally absolutely continuous in $I$, locally uniform with respect to $\mathcal{U}_{r_{3}}$.

The function $p(z, t)$ defined by

$$
p(z, t)=z \frac{\partial \mathcal{L}(z, t)}{\partial z} / \frac{\partial \mathcal{L}(z, t)}{\partial t}
$$

is analytic in a $\operatorname{disc} \mathcal{U}_{r}, 0<r<r_{3}$, for all $t \in I$.

If the function

$$
w(z, t)=\frac{p(z, t)-1}{p(z, t)+1}=\frac{\frac{z \partial \mathcal{L}(z, t)}{\partial z}-\frac{\partial \mathcal{L}(z, t)}{\partial t}}{\frac{z \partial \mathcal{L}(z, t)}{\partial z}+\frac{\partial \mathcal{L}(z, t)}{\partial t}}
$$


is analytic in $\mathcal{U} \times I$ and $|w(z, t)|<1$ for all $z \in \mathcal{U}$ and $t \in I$, then $p(z, t)$ has an analytic extension with a positive real part in $\mathcal{U}$ for all $t \in I$. We take $\zeta$ instead of $z$ in equality (3.6); then we have

$$
w(\zeta, t)=\frac{(1+s) \mathcal{G}(\zeta, t)-2}{(1-s) \mathcal{G}(\zeta, t)+2}
$$

where

$$
\mathcal{G}(\zeta, t)=\frac{1}{m s} \frac{e^{s t} \zeta f^{\prime}\left(e^{s t} \zeta\right)}{f\left(e^{s t} \zeta\right) g\left(e^{s t} \zeta\right)}-2 t \frac{e^{s t} \zeta g^{\prime}\left(e^{s t} \zeta\right)}{g\left(e^{s t} \zeta\right)}
$$

for $\zeta \in \mathcal{U}^{*}$ and $t \in I$.

The inequality $|w(\zeta, t)|<1$ for all $\zeta \in \mathcal{U}^{*}$ and $t \in I$ is equivalent to

$$
\left|\mathcal{G}(\zeta, t)-\frac{1}{\alpha}\right|<\frac{1}{\alpha}, \quad \zeta \in \mathcal{U}^{*}, t \in[0, \infty), \alpha=\Re(s)
$$

where $w(\zeta, t)$ is defined by (3.7).

Define

$$
\mathcal{H}(\zeta, t)=\mathcal{G}(\zeta, t)-\frac{1}{\alpha}, \quad \zeta \in \mathcal{U}^{*}, t \in[0, \infty), \alpha=\Re(s) .
$$

From (3.1), (3.8) and (3.10), we have

$$
|\mathcal{H}(\zeta, 0)|=\left|\frac{\zeta f^{\prime}(\zeta)}{f(\zeta) g(\zeta)}-\frac{m s}{\alpha}\right|<\frac{m|s|}{\alpha}
$$

Since $\left|\frac{e^{s t}}{\zeta}\right| \geq\left|e^{s t}\right|=e^{\alpha t}>1$ for all $z \in \overline{\mathcal{U}}=\{z \in \mathbb{C}:|z| \leq 1\}$ and $t>0$, we find that $\mathcal{H}(\zeta, t)$ is an analytic function in $\overline{\mathcal{U}^{*}}$. Using the maximum modulus principle, it follows that for all $\zeta \in \mathcal{U}^{*}$ and each $t>0$, arbitrarily fixed, there exists $\theta=\theta(t) \in \mathbb{R}$ such that

$$
|\mathcal{H}(\zeta, t)|<\max _{|\zeta|=1}|\mathcal{H}(\zeta, t)|=\left|\mathcal{H}\left(e^{i \theta}, t\right)\right|
$$

for all $\zeta \in \mathcal{U}^{*}$ and $t \in I$.

Denote $u=e^{s t} e^{-i \theta}$. Then $|u|=e^{\alpha t}$, and from (3.8) we have

$$
\left|\mathcal{H}\left(e^{i \theta}, t\right)\right|=\left|\frac{u f^{\prime}(u)}{f(u) g(u)}-\left(2 \ln |u| \frac{u g^{\prime}(u)}{g(u)}+1\right) \frac{m s}{\alpha}\right| .
$$

Because $u \in \mathcal{U}^{*}$, inequality (3.2) implies that

$$
\left|\mathcal{H}\left(e^{i \theta}, t\right)\right| \leq \frac{m|s|}{\alpha},
$$

and from (3.11), (3.13) and (3.12), we conclude that

$$
|\mathcal{H}(\zeta, t)|=\left|\mathcal{G}(\zeta, t)-\frac{1}{\alpha}\right|<\frac{1}{\alpha}
$$

for all $\zeta \in \mathcal{U}^{*}$ and $t \in I$. Therefore $|w(z, t)|<1$ for all $z \in \mathcal{U}$ and $t \in I$. 
Since all the conditions of Theorem 2.1 are satisfied, we obtain that the function $\mathcal{L}(z, t)$ has an analytic and univalent extension to the whole unit $\operatorname{disc} \mathcal{U}$, for all $t \in I$. For $t=0$, we have $\mathcal{L}(z, 0)=1 / f\left(z^{-1}\right)$, for $z \in \mathcal{U}$ and therefore the function $f(\zeta)$ is univalent in $\mathcal{U}^{*}$.

If we take $g(\zeta)=\frac{\zeta f^{\prime}(\zeta)}{f(\zeta)}$ in Theorem 3.1, then we have the following result.

Corollary 3.2 Let $m \in \mathbb{R}^{+}$and $s$ be a complex number such that $s=\alpha+i \beta, \alpha>0, \beta \in \mathbb{R}$; $1 / 2<m \leq \alpha$ and $f \in \Sigma, f^{\prime}(\zeta) \neq 0$. If the inequality

$$
\left|1-\left\{2 \ln |\zeta|\left(1+\frac{\zeta f^{\prime \prime}(\zeta)}{f^{\prime}(\zeta)}-\frac{\zeta f^{\prime}(\zeta)}{f(\zeta)}\right)+1\right\} \frac{m s}{\alpha}\right| \leq \frac{m|s|}{\alpha}
$$

holds true for all $\zeta \in \mathcal{U}^{*}$, then the function $f$ is univalent in $\mathcal{U}^{*}$.

For $g(\zeta)=\frac{\zeta}{f(\zeta)}$ in Theorem 3.1, we have the following corollary.

Corollary 3.3 Let $m \in \mathbb{R}^{+}$and s be a complex number such that $s=\alpha+i \beta, \alpha>0, \beta \in \mathbb{R}$; $1 / 2<m \leq \alpha$ and $f \in \Sigma, f^{\prime}(\zeta) \neq 0$. If the inequalities

$$
\left|f^{\prime}(\zeta)-\frac{m s}{\alpha}\right| \leq \frac{m|s|}{\alpha}
$$

and

$$
\left|f^{\prime}(\zeta)-\left\{2 \ln |\zeta|\left(1-\frac{\zeta f^{\prime}(\zeta)}{f(\zeta)}\right)+1\right\} \frac{m s}{\alpha}\right| \leq \frac{m|s|}{\alpha}
$$

hold true for all $\zeta \in \mathcal{U}^{*}$, then the function $f$ is univalent in $\mathcal{U}^{*}$.

Putting $g(\zeta)=1$ in Theorem 3.1, we obtain a simple univalence condition as follows.

Corollary 3.4 Let $m \in \mathbb{R}^{+}$and s be a complex number such that $s=\alpha+i \beta, \alpha>0, \beta \in \mathbb{R}$; $1 / 2<m \leq \alpha$ and $f \in \Sigma, f^{\prime}(\zeta) \neq 0$. If the inequality

$$
\left|\frac{\zeta f^{\prime}(\zeta)}{f(\zeta)}-\frac{m s}{\alpha}\right| \leq \frac{m|s|}{\alpha}
$$

holds true for all $\zeta \in \mathcal{U}^{*}$, then the function $f$ is univalent in $\mathcal{U}^{*}$.

\section{Quasiconformal extension criterion}

In this section we will refine the univalence condition given in Theorem 3.1 to a quasiconformal extension criterion.

Theorem 4.1 Let $m \in \mathbb{R}^{+}$and $s$ be a complex number such that $s=\alpha+i \beta, \alpha>0, \beta \in \mathbb{R}$; $1 / 2<m \leq \alpha ; k \in[0,1)$ and $f \in \Sigma, f^{\prime}(\zeta) \neq 0$. If there exists an analytic function $g$ in $\mathcal{U}^{*}$ such that $g(\zeta)=1+c_{2} \zeta^{-2}+\cdots$ and the inequalities

$$
\left|\frac{\zeta f^{\prime}(\zeta)}{f(\zeta) g(\zeta)}-\frac{m s}{\alpha}\right|<k \frac{m|s|}{\alpha}
$$


and

$$
\left|\frac{\zeta f^{\prime}(\zeta)}{f(\zeta) g(\zeta)}-\left(2 \ln |\zeta| \frac{\zeta g^{\prime}(\zeta)}{g(\zeta)}+1\right) \frac{m s}{\alpha}\right| \leq k \frac{m|s|}{\alpha}
$$

hold true for all $\zeta \in \mathcal{U}^{*}$, then the function $f$ has a l-quasiconformal extension to $\mathbb{C}$, where

$$
l=\frac{|s-1|^{2}+k\left|(\bar{s})^{2}-1\right|}{\left|(\bar{s})^{2}-1\right|+k|s-1|^{2}}<1 .
$$

Proof In the proof of Theorem 3.1, it has been shown that the function $\mathcal{L}(z, t)$ given by (3.3) is a subordination chain in $\mathcal{U}$. Applying Theorem 2.2 to the function $w(\zeta, t)$ given by (3.7), we obtain that the condition

$$
\left|\frac{(1+s) \mathcal{G}(\zeta, t)-2}{(1-s) \mathcal{G}(\zeta, t)+2}\right|<l, \quad \zeta \in \mathcal{U}^{*}, t \geq 0 \text { and } l \in[0,1)
$$

implies $l$-quasiconformal extensibility of $f$, where $\mathcal{G}(\zeta, t)$ is defined by (3.8).

Lengthy but elementary calculation shows that the last inequality (4.3) is equivalent to

$$
\left|\mathcal{G}(\zeta, t)-\frac{2\left(\left(1+l^{2}\right)+\alpha\left(1-l^{2}\right)\right)-2 \beta\left(1-l^{2}\right) i}{2 \alpha\left(1+l^{2}\right)+\left(1-l^{2}\right)\left(1+|s|^{2}\right)}\right| \leq \frac{4 l}{2 \alpha\left(1+l^{2}\right)+\left(1-l^{2}\right)\left(1+|s|^{2}\right)}
$$

It is easy to check that, under the assumptions (4.1) and (4.2), we have

$$
\left|\mathcal{G}(\zeta, t)-\frac{1}{\alpha}\right| \leq \frac{k}{\alpha}
$$

Consider the two discs $\Delta$ and $\Delta^{\prime}$ defined by (4.4) and (4.5) respectively, where $\mathcal{G}(\zeta, t)$ is replaced by a complex variable $w$. Our theorem will be proved if we find the smallest $l \in[0,1)$ for which $\Delta^{\prime}$ is contained in $\Delta$. This will be done if and only if the distance apart of the centers plus the smallest radius is equal, at most, to the largest radius. So, we are required to prove that

$$
\left|\frac{2\left(\left(1+l^{2}\right)+\alpha\left(1-l^{2}\right)\right)-2 \beta\left(1-l^{2}\right) i}{2 \alpha\left(1+l^{2}\right)+\left(1-l^{2}\right)\left(1+|s|^{2}\right)}-\frac{1}{\alpha}\right|+\frac{k}{\alpha} \leq \frac{4 l}{2 \alpha\left(1+l^{2}\right)+\left(1-l^{2}\right)\left(1+|s|^{2}\right)}
$$

or equivalently,

$$
\frac{\left(1-l^{2}\right)\left|(\bar{s})^{2}-1\right|}{2 \alpha\left[2 \alpha\left(1+l^{2}\right)+\left(1-l^{2}\right)\left(1+|s|^{2}\right)\right]} \leq \frac{2 l}{2 \alpha\left(1+l^{2}\right)+\left(1-l^{2}\right)\left(1+|s|^{2}\right)}-\frac{k}{2 \alpha}
$$

with the condition

$$
\frac{2 l}{2 \alpha\left(1+l^{2}\right)+\left(1-l^{2}\right)\left(1+|s|^{2}\right)}-\frac{k}{2 \alpha} \geq 0
$$

Now we will solve inequalities (4.6) and (4.7). If in (4.6) the inequality sign is replaced by equal, making use of Mathematica program, we obtain the following two solutions:

$$
L_{1}=\frac{|s-1|^{2}+k\left|(\bar{s})^{2}-1\right|}{\left|(\bar{s})^{2}-1\right|+k|s-1|^{2}}, \quad L_{2}=-\frac{|s+1|^{2}+k\left|(\bar{s})^{2}-1\right|}{\left|(\bar{s})^{2}-1\right|+k|s-1|^{2}} .
$$


Therefore, the solution of inequality (4.6) is $l \leq L_{2}$ and $L_{1} \leq l$. Since $L_{2}<0$, it remains $L_{1} \leq l$.

After similar calculations, from inequality (4.7), we have $l \leq \mathcal{L}_{2}$ and $\mathcal{L}_{1} \leq l$, where

$$
\begin{aligned}
& \mathcal{L}_{1}=\frac{-2 \alpha+\sqrt{4 \alpha^{2}+\left|(\bar{s})^{2}-1\right|^{2} k^{2}}}{k|s-1|^{2}}, \\
& \mathcal{L}_{2}=\frac{-2 \alpha-\sqrt{4 \alpha^{2}+\left|(\bar{s})^{2}-1\right|^{2} k^{2}}}{k|s-1|^{2}} .
\end{aligned}
$$

Since $\mathcal{L}_{2}<0$, we get $\mathcal{L}_{1} \leq l$.

Again, making use of Mathematica program, we obtain $\mathcal{L}_{1} \leq L_{1}$. Therefore $L_{1} \leq l<1$ and the proof is complete.

\section{Competing interests}

The authors declare that they have no competing interests.

\section{Authors' contributions}

All authors read and approved the final manuscript.

\section{Acknowledgements}

Dedicated to Professor Hari M Srivastava.

The authors thank the referees for their valuable suggestions to improve the paper. The present investigation was supported by Atatürk University Rectorship under 'The Scientific and Research Project of Atatürk University', Project No: 2012/173.

Received: 10 November 2012 Accepted: 26 February 2013 Published: 19 March 2013

\section{References}

1. Becker, J: Löwnersche differential gleichung und quasikonform fortsetzbare schlichte functionen. J. Reine Angew. Math. 255, 23-43 (1972) (in German)

2. Becker, J: Über die Lösungsstruktur einer Differentialgleichung in der Konformen Abbildung. J. Reine Angew. Math. 285, 66-74 (1976)

3. Ahlfors, LV: Sufficient conditions for quasiconformal extension. Ann. Math. Stud. 79, 23-29 (1974)

4. Lewandowski, Z: On a univalence criterion. Bull. Acad. Pol. Sci., Sér. Sci. Math. 29, 123-126 (1981)

5. Lewandowski, Z: Some remarks on univalence criteria. Ann. Univ. Mariae Curie-Sklodowska, Sect. A 36/37, 87-95 (1982/1983)

6. Miazga, J, Wesolowski, A: A univalence criterion for meromorphic functions. Ann. Pol. Math. 56(1), 63-66 (1991)

7. Ruscheweyh, S: An extension of Becker's univalence condition. Math. Ann. 220, 285-290 (1976)

8. Singh, V, Chichra, PN: An extension of Becker's criterion for univalence. J. Indian Math. Soc. 41, 353-361 (1977)

9. Deniz, E: Sufficient conditions for univalence and quasiconformal extensions of meromorphic functions. Georgian Math. J. 19(4), 639-653 (2012)

10. Deniz, E, Orhan, H: Univalence criterion for analytic functions. Gen. Math. 4, 211-220 (2009)

11. Deniz, E, Orhan, H: Univalence criterion for meromorphic functions and Loewner chains. Appl. Math. Comput. 218(6), 751-755 (2011)

12. Ponnusamy, S, Sugawa, T: Norm estimates and univalence criteria for meromorphic functions. J. Korean Math. Soc. 45(6), 1661-1676 (2008)

13. Răducanu, D, Orhan, H, Deniz, E: On some sufficient conditions for univalence. An. Univ. 'Ovidius' Constanţa, Ser. Mat. $18(2), 217-222(2010)$

14. Kanas, S, Lecko, A: Univalence criteria connected with arithmetic and geometric means, II. Zeszyty Nauk. Politech. Rzeszowskiej Mat. 20, 49-59 (1996)

15. Kanas, S, Lecko, A: Univalence criteria connected with arithmetic and geometric means, II. In: Proceedings of the Second Int. Workshop of Transform Methods and Special Functions, Varna'96, pp. 201-209. Bulgar Acad. Sci., Sofia (1996)

16. Kanas, S, Srivastava, HM: Some criteria for univalence related to Ruscheweyh and Salagean derivatives. Complex Var. Elliptic Equ. 38, 263-275 (1997)

17. Pommerenke, C: Über die Subordination analytischer Funktionen. J. Reine Angew. Math. 218, 159-173 (1965)

18. Pommerenke, C: Univalent Functions. Vandenhoeck Ruprecht, Göttingen (1975)

19. Becker, J: Conformal mappings with quasiconformal extensions. In: Brannan, DA, Clunie, JG (eds.) Aspects of Contemporary Complex Analysis, pp. 37-77. Academic Press, San Diego (1980)

20. Anderson, JM, Hinkkanen, A: Univalence criteria and quasiconformal extensions. Trans. Am. Math. Soc. 324, 823-842 (1991)

21. Betker, Th: Löewner chains and quasiconformal extensions. Complex Var. 20, 107-111 (1992)

22. Pfaltzgraff, JA: k-quasiconformal extension criteria in the disk. Complex Var. 21, 293-301 (1993) 
23. Hotta, I: Löwner chains with complex leading coefficient. Monatshefte Math. 163(3), 315-325 (2011)

24. Hotta, I: Explicit quasiconformal extensions and Loewner chains. Proc. Jpn. Acad., Ser. A, Math. Sci. 85, 108-111 (2009)

25. Hotta, I: Löewner chains and quasiconformal extension of univalent functions, Dissertation, Tohoku Univ., 2010

doi:10.1186/1029-242X-2013-112

Cite this article as: Çağlar and Orhan: Univalence criteria for meromorphic functions and quasiconformal extensions. Journal of Inequalities and Applications 2013 2013:112.

Submit your manuscript to a SpringerOpen ${ }^{\circ}$ journal and benefit from:

- Convenient online submission

- Rigorous peer review

- Immediate publication on acceptance

Open access: articles freely available online

- High visibility within the field

- Retaining the copyright to your article

Submit your next manuscript at $>$ springeropen.com 